\title{
Coeficiente del tanque evaporímetro Clase A para estimar la evapotranspiración de referencia para el valle de Tumbaco
}

\section{The Class A pan coefficient to estimate the reference evapotranspiration for the Tumbaco valley}

\author{
Randon Ortiz C. ${ }^{1}$, Christian Tamayo O. ${ }^{1}$, Maritza Chile A. ${ }^{1}$, Andrés Méndez Ch. \\ 凶: rsortiz@uce.edu.ec \\ 1 Carrera Ingeniería Agronómica, Facultad de Ciencias Agrícolas, Universidad Central del Ecuador, \\ 2 Instituto Nacional de Meteorología e Hidrología, Quito, Ecuador.
}

\begin{abstract}
Resumen
La determinación precisa de los coeficientes del tanque evaporímetro Clase A permiten estimar la evapotranspiración de referencia y obtener valores reales de necesidades hídricas de los cultivos. Se utilizó información climatológica diaria y mensual para el análisis del coeficiente del tanque $(\mathrm{Kp})$ y de la evapotranspiración de referencia (ET). Los métodos utilizados para determinar Kp fueron: Allen - Pruitt, Cuenca, Pereira, Snyder y el coeficiente calculado. Se determinaron los parámetros inferenciales para el análisis cruzado entre los diferentes métodos para estimar ET: error medio absoluto, coeficiente de concordancia y el coeficiente de determinación. Los resultados demuestran que: i) para información climatológica diaria, el contraste entre los métodos Allen - Pruitt y Snyder presentó los índices inferenciales de mayor precisión; ii) para información del clima mensual, el contraste Snyder - Coeficiente calculado presentó índices de mayor precisión, siendo 0.72 el coeficiente Kp promedio; iii) la máxima $\mathrm{ET}_{\mathrm{o}}$ promedio se registró en el mes de agosto con $139.32 \mathrm{~mm}$ y la mínima en abril con $76.54 \mathrm{~mm}$. Las conclusiones principales son: i) los métodos Snyder y el coeficiente calculado pueden utilizarse para determinar el coeficiente Kp para información climatológica mensual y el método Snyder para información diaria y ii) el método estándar Penman Monteith FAO 56 presentó índices inferenciales bajos para información climática diaria y de mayor precisión para información promedio mensual, considerando el entorno agroecológico del valle de Tumbaco.
\end{abstract}

Palabras clave: Ecuador, Penman Monteith, evaporación, necesidades hídricas de los cultivos, balance hídrico.

\begin{abstract}
The precise determination of the Class A pan coefficients allows to estimate the reference evapotranspiration and real values of crop water requirements. Daily and monthly climatological data was used to determine the pan coefficient $(\mathrm{Kp})$ and the reference evapotranspiration $\left(\mathrm{ET}_{\mathrm{o}}\right)$. The models evaluated to determine Kp were: Allen - Pruit, Cuenca, Pereira, Snyder and the computed coefficient. The following indices were used to evaluate the cross analysis between the models to compute $\mathrm{ET}_{\mathrm{o}}$ : absolute mean error, concordance coefficient and the coefficient of determination. The results show that: i) for daily data, the Allen - Pruitt and Snyder contrast presented the best indices; ii) for monthly data, the Snyder and the computed coefficient contrast presented indices with higher accuracy; iii) the maximum average ET was registered in August with $139.32 \mathrm{~mm}$ and the minimum in April with about $76.54 \mathrm{~mm}$. The main conclusions are: i) for monthly average climatological data, the Snyder and the computed method can be used to determine Kp and the Snyder method for daily data and ii) the standard Penman Monteith FAO 56 method presented poor inferential indices for daily data and better indices for monthly data, considering the environmental conditions of the Tumbaco valley.
\end{abstract}

Keywords: Ecuador, Penman Monteith, evaporation, crop water requirements, water budget. 


\section{Introducción}

La planificación del manejo del agua en un sistema de riego es de vital importancia en una agricultura sostenible. La cuantificación mensual o decadal de la disponibilidad de los recursos hídricos se determina a partir de un balance hídrico, en el cual, un valor positivo representa un exceso de agua (drenaje) y un valor negativo un déficit (riego). El diseño de la infraestructura y la aplicación del agua de riego se determinan con base en las necesidades hídricas de los cultivos, en donde, la evapotranspiración de referencia es una variable muy importante. Una sobrestimación de la ET influirá en un sobredimensionamiento de la infraestructura de riego y en una entrega excesiva del agua, por el contrario, una subestimación de la $\mathrm{ET}_{\mathrm{o}}$, influirá en el diseño de una infraestructura de riego que no permitirá cubrir la demanda de agua de los cultivos.

La $\mathrm{ET}_{\mathrm{o}}$ puede determinarse por varios métodos (directos e indirectos), de entre los cuales, en el presente estudio, se aborda el método estándar Penman Monteith FAO 56 (FAO56) y el tanque evaporímetro Clase A (Allen et al., 2006). La Organización de las Naciones Unidas para la Agricultura y la Alimentación (FAO), recomienda utilizar el método estándar FAO56 para determinar $\mathrm{ET}_{\mathrm{o}}$, debido a que se ajusta tanto para zonas áridas como templadas o tropicales (Allen et al., 2006). El tanque evaporímetro Clase A fue desarrollado en los Estados Unidos por el Servicio Nacional del Clima (Doorenbos \& Pruitt, 1977), es muy utilizado en zonas agrícolas para planificar la aplicación del agua de riego, debido a que mide el efecto integrado del clima, es de fácil lectura y es muy económico (Allen et al., 2006). Para estimar $\mathrm{ET}_{\mathrm{o}}$ a partir del tanque evaporímetro se necesita conocer el coeficiente del tanque, el cual puede determinarse a través de varios métodos como: AllenPruitt, Cuenca, Pereira, Snyder y a través de dividir ET $_{\text {o }}$ para la evaporación (Doorenbos \& Pruitt, 1977; Grismer et al., 2002; Allen et al., 2006). Según FAO (para condiciones ambientales mundiales promedio), el coeficiente Kp varía entre 0.40 y 0.85 (Allen et al., 2006), en tanto que, para el Servicio Nacional del Clima (para el territorio de los Estados Unidos), el coeficiente Kp varía entre 0.40 y 0.87 (Grismer et al., 2002). En la región, para condiciones ambientales de Sao Paulo (Brasil), el coeficiente Kp promedio fue 0.71 (Sentelhas \& Folegatti, 2003); para la zona sur del Uruguay, el coeficiente Kp promedio fue 0.71 (Pupo \& García, 2009) y para algunos sitios del Perú, este coeficiente varió entre 0.78 y 0.85 (Hamilton, 2013). Según Doorenbos \& Pruitt (1977), para una humedad relativa mayor al $70 \%$ y una velocidad del viento comprendida entre 175 y $425 \mathrm{Km} \mathrm{d}^{-1}$, condiciones ambientales del valle de Tumbaco, el coeficiente Kp varía entre 0.65 y 0.75 .

Allen et al. (1990), realizó un estudio para determinar $\mathrm{ET}_{\mathrm{o}}$ por varios métodos para condiciones ambientales de Kimberly (Idaho), tomando como base $\mathrm{ET}_{\mathrm{o}}$ determinada a través de lisímetros, encontrando que el método Penman Monteith presentó los índices inferenciales de mayor precisión, mientras que el método del tanque evaporímetro se ubicó en el puesto 14 de los 20 métodos estudiados; Sentelhas \& Folegatti (2003), para condiciones ambientales de Sao Paulo (Brasil) y con base en lisímetros, encontraron que la $\mathrm{ET}_{\mathrm{o}}$ determinada a través del tanque evaporímetro con los coeficientes Pereira y Cuenca presentaron índices de mayor precisión que Penman Monteith FAO56.

La programación del riego (tiempo y frecuencia de aplicación), se realiza con base en las necesidades hídricas de los cultivos, requiriéndose determinar la $\mathrm{ET}_{\mathrm{o}}$ con precisión, especialmente para operar sistemas de riego por surcos o aspersión. En el Centro Académico Docente Experimental La Tola (CADET) se producen cultivos comerciales y se desarrollan proyectos de investigación de diverso tipo y no se cuenta con un balance hídrico actualizado que facilite programar el riego, siendo necesario determinar $\mathrm{ET}_{\text {。 }}$ para estimar las necesidades hídricas de los cultivos.

El objetivo del estudio consistió en evaluar diferentes métodos para determinar el coeficiente del tanque (Kp) de mejor ajuste para estimar $\mathrm{ET}_{\mathrm{o}}$ utilizando el método del tanque evaporímetro clase A.

\section{Materiales y métodos}

El sitio experimental para la presente investigación se estableció en el Valle de Tumbaco, localizado en la República del Ecuador, provincia de Pichincha. Se utilizó información climatológica de la estación La Tola (M002) perteneciente al Instituto Nacional de Meteorología e Hidrología (INAMHI), ubicada en la cuenca alta del río Esmeraldas, a una altura de $2.480 \mathrm{~m}$, a $00^{\circ} 13^{\prime} 46^{\prime \prime}$ de latitud sur y $78^{\circ} 22^{\prime} 0^{\prime \prime}$ de longitud oeste; los intervalos de tiempo considerados para el análisis fueron a nivel diario (datos de enero a diciembre de 2017) y mensuales (datos desde 1986 hasta 2013).

Las condiciones agroecológicas del valle de Tumbaco se caracterizan por tener dos estaciones climatológicas bien diferenciadas, la estación lluvio- 
sa de octubre a mayo y verano de junio a septiembre, históricamente, agosto es el mes más seco, con una mayor tasa de evaporación, menor humedad relativa y menor precipitación.

Tabla 1. Información climatológica estación la Tola. Serie mensual 1986 - 2013.

\begin{tabular}{lccc}
\hline \multicolumn{1}{c}{ Variable } & Máximo & Promedio & Mínimo \\
\hline Humedad relativa $(\%)$ & 86.0 & 74.6 & 57.0 \\
Velocidad viento $\left(\mathrm{m} \mathrm{s}^{-1}\right)$ & 13.9 & 2.7 & 0.1 \\
Temperatura máxima $\left({ }^{\circ} \mathrm{C}\right)$ & 29.5 & 25.8 & 22.5 \\
Temperatura mínima $\left({ }^{\circ} \mathrm{C}\right)$ & 9.2 & 5.9 & 1.6 \\
Horas de luz diarias & 9.2 & 5.7 & 2.8 \\
Evaporación mensual $(\mathrm{mm})$ & 293.6 & 134.0 & 69.3 \\
Precipitación anual $(\mathrm{mm})$ & 1179.8 & 853.7 & 553.3 \\
\hline
\end{tabular}

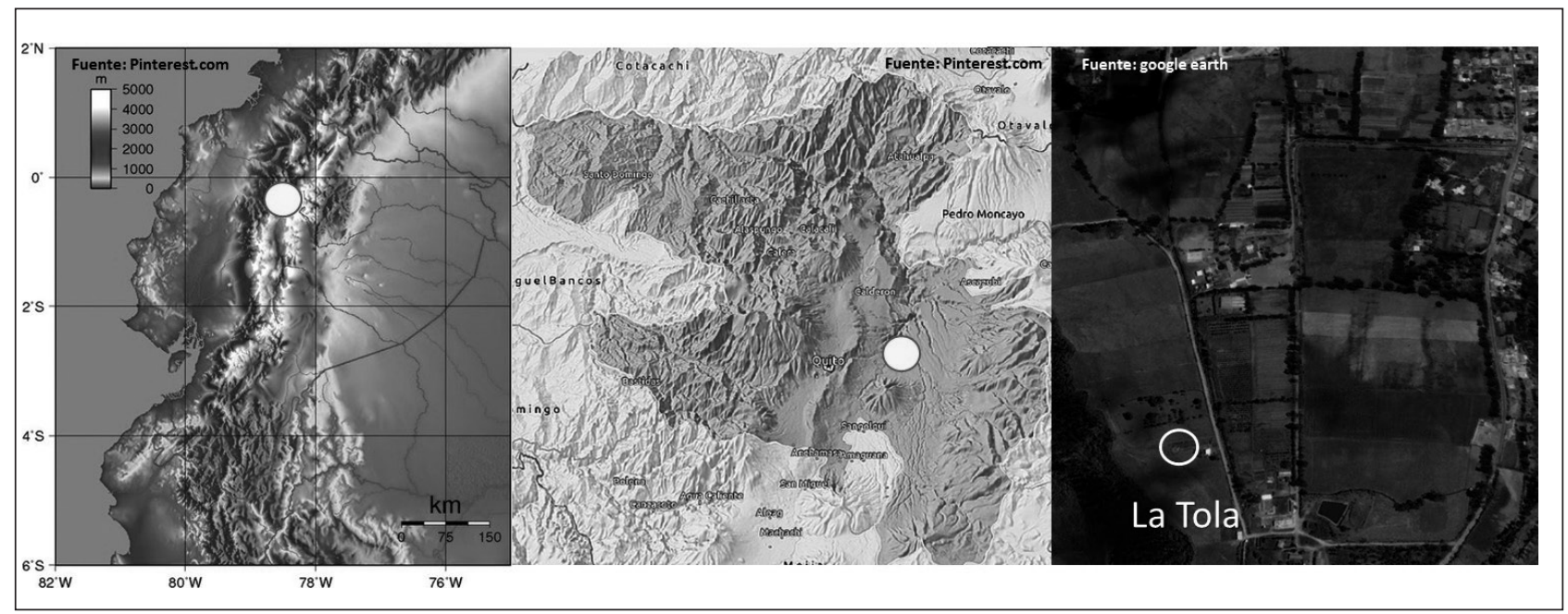

Figura 1. Ubicación de la estación climatológica La Tola.

\subsection{Métodos para determinar la evapotranspira- ción de referencia}

Penman Monteith FAO 56, determina ET con base en tres componentes: i) resistencia aerodinámica (demanda evaporativa de la atmósfera estimada en función de las variables climatológicas: temperatura promedio, máxima y mínima, humedad relativa, horas de luz solar, viento, altitud y latitud); ii) resistencia superficial del cultivo (flujo del agua por difusión desde las raíces hasta los estomas de la planta y de la evaporación directa del agua desde el suelo) y iii) albedo (radiación solar reflejada por el cultivo); para el cultivo de referen- cia (pasto) de una altura de $0.12 \mathrm{~m}$, bien regado y con una cobertura total de la superficie del suelo, la resistencia superficial estimada es de $70 \mathrm{~s} \mathrm{~m}^{-1}$ y la radiación solar reflejada o albedo es 0.23 (Allen et al., 2006).

$E T_{o}=\frac{0.408 \times \Delta \times(R n-G)+\gamma \times \frac{900}{(T+273)} \times U_{2} \times(e s-e a)}{\Delta+\gamma \times\left(1+0.34 \times U_{2}\right)}$

En donde: $E T_{\text {o }}$ es la evapotranspiración referencia $\left(\mathrm{mm} \mathrm{d}^{-1}\right) ; R n$, radiación neta en la superficie del cultivo ( $\mathrm{MJ} \mathrm{m}^{-2} \mathrm{~d}^{-1}$ ); $G$, flujo del calor de suelo $\left(\mathrm{MJ} \mathrm{m}^{-2} \mathrm{~d}^{-1}\right) ; U_{2}$, velocidad del viento $\left(\mathrm{m} \mathrm{s}^{-1}\right) ; e a$, 
presión real de vapor $(\mathrm{kPa})$; es, presión de vapor de saturación; $\Delta$, pendiente de la curva de variación de la presión $\left(\mathrm{kPa}^{\circ} \mathrm{C}^{-1}\right)$; $T$, temperatura promedio $\left({ }^{\circ} \mathrm{C}\right)$; $\gamma$, calor latente de vaporización $\left(\mathrm{kPa}^{\circ} \mathrm{C}^{-1}\right)$.

Tanque evaporímetro Clase $A$, proporciona una medida integrada del efecto de la radiación, viento, temperatura y humedad sobre el proceso evaporativo que ocurre en una superficie libre de agua, a pesar de que existen varios factores que difieren entre la evaporación y la transpiración, como: reflexión de la radiación solar, almacenamiento de calor en el agua contenida en el tanque, turbulencia alrededor del tanque, transferencia de calor a través de las paredes del tanque y localización (variables que influyen en el balance energético), el tanque evaporímetro puede utilizarse para estimar ET en forma confiable cuando las lecturas de la lámina de agua se realizan correctamente. $\mathrm{La} \mathrm{ET}_{\mathrm{o}}$ se determina a través de la utilización de un coeficiente empírico derivado de las condiciones físicas y ambientales que rodean al tanque, el cual varía entre 0.40 y 0.85 (Allen et al., 2006).

$$
E T_{o}=K p \times E o
$$

Donde: $K p$ es el coeficiente del tanque evaporímetro y Eo, la evaporación del tanque $\left(\mathrm{mm} \mathrm{d}^{-1}\right)$.

\subsection{Métodos para determinar el coeficiente del tanque evaporímetro}

Allen - Pruitt, desarrollado a partir de la interpolación de los coeficientes del tanque publicados por el Servicio Nacional del Clima (NWS), cuyos coeficientes se calibraron con base en lisímetros instalados en todo el territorio de los Estados Unidos (Grismer et al., 2002).

$$
K p \approx 0.108-0.000331 \times U_{2}+0.0422 \times \operatorname{Ln}(F)+0.1434 \times \operatorname{Ln}(H R)-0.000631 \times(\operatorname{Ln}(F))^{2} \times \operatorname{Ln}(H R)
$$

Donde: $F$ es la distancia que existe entre el tanque evaporímetro y el área con cultivo (m), medido igual que $1.5 \mathrm{~m} ; H R$, la humedad relativa (\%).

Cuenca, desarrollado a partir de la interpolación de los coeficientes del tanque publicados por FAO-24 (Bhabagrahi et al., 2012).

$$
\begin{aligned}
& -2.45 \times 10^{-4} \times U_{2}+5.16 \times 10^{-3} \times H R+1.18 \times 10^{-3}-1.6 \times 10^{-5} \times H R^{2} \\
& \times F^{2}-8 \times 10^{-9} \times H R^{2} \times U_{2}-1 \times 10^{-8} \times H R^{2} \times F
\end{aligned}
$$

Pereira, desarrollado para condiciones ambientales de Brasil (Pereira et al., 1995).

$$
K p=0.51206-0.000321 \times U_{2}+0.002889 \times H R+0.031886 \times \operatorname{Ln}(F)-0.000107 \times H R \times \operatorname{Ln}(F)
$$

Snyder, desarrollado a partir de la interpolación de los coeficientes del tanque publicados por NWS (Snyder et al., 2005).

$$
K p=0.5321-3 \times 10^{-4} \times U_{2}+0.0249 \times \operatorname{Ln}(F)+0.0025 \times H R
$$


Kp Calculado, cociente de dividir la evapotranspiración de referencia (FAO56) para la evaporación del tanque (Allen et al., 2006).

Abreviaciones: ET determinada con el método Penman Monteith FAO 56 se define como FAO56; ET determinada a través del tanque evaporímetro como Tan; el método Allen -Pruitt para calcular el coeficiente del tanque se define como AP, Cuenca como C, Pereira como $\mathrm{P}$, Snyder como $\mathrm{S}$ y el coeficiente calculado como $\mathrm{Kpc}$; $\mathrm{ET}_{\mathrm{o}}$ determinada con el coeficiente del tanque: Allen - Pruitt como Tan-AP, Cuenca Tan-C, Pereira Tan-P, Snyder Tan-S y el calculado como Tan-Kpc.

\subsection{Parámetros inferenciales de evaluación}

Se realizó un análisis cruzado entre las diferentes metodologías para determinar el método de mayor precisión para calcular el coeficiente del tanque, basado en los siguientes índices inferenciales:

Error medio absoluto (MAE), utilizado para establecer la diferencia que existe entre una variable medida con relación a una estimada, es el promedio de todos los errores absolutos; cuando este valor tiende a cero, el error es menor entre la variable medida y la estimada, el valor óptimo es igual a cero (Chai, 2014).

$$
M A E=\frac{\sum_{i=1}^{n} O_{i}-E_{i}}{n}
$$

Donde: $O$, es el valor observado; $E$, el valor proyectado; $n$, el número de valores en estudio.

Coeficiente de determinación $\left(R^{2}\right)$, calculado a través de regresiones simples, es un índice estadístico utilizado para medir la relación lineal entre dos variables cuantitativas; el valor óptimo debe ser igual a uno y cuando este valor tiende a cero, la correlación entre dos variables es baja; cuando el valor es positivo, la relación es directa y cuando el valor es negativo, la relación es inversa (Samuels, 2014).

$$
R^{2}=\frac{\left[\sum_{i=1}^{n}\left(O_{i}-X\right)\left(E_{i}-E_{m}\right)\right]^{2}}{\left(\sum_{i=1}^{n} O i^{2}-n X^{2}\right) \times\left(\sum_{i=1}^{n} E i^{2}-n E_{m}^{2}\right)}
$$

Donde: $E i$, es el valor proyectado; $E m$, el promedio de los valores proyectados; $X$, el promedio de los valores observados.

Coeficiente de concordancia $(D)$, determina la precisión de los resultados proporcionados por el modelo utilizado para pronosticar un valor ob- servado; cuando este valor tiende a uno, el modelo presenta una mayor precisión (Willmott et al., 2011)

$$
D=1-\frac{\sum_{i=1}^{n}\left(O_{i}-E_{i}\right)^{2}}{\sum_{i=1}^{n}\left(O_{i}-X+E_{i}-X\right)^{2}}
$$

\section{Resultados}

\subsection{Coeficiente del tanque para información clima- tológica diaria}

En relación al coeficiente $\mathrm{Kp}$, se observó que éste no es constante y varía en función de los pará metros climatológicos (humedad relativa y viento). El coeficiente calculado proporcionó los valores más altos (Figura 2b), seguido por los métodos Pereira, Cuenca, Allen - Pruitt y Snyder (Figura 2a). 
Tabla 2. Coeficientes Kp para información climatológica diaria y mensual.

\begin{tabular}{cccccccc}
\hline & & \multicolumn{3}{c}{ Datos diarios } & \multicolumn{3}{c}{ Datos mensuales } \\
\cline { 3 - 7 } No & Método & Máximo & Promedio & Mínimo & Máximo & Promedio & Mínimo \\
\hline 1 & Allen - Pruitt & 0.78 & 0.75 & 0.68 & 0.76 & 0.74 & 0.70 \\
2 & Cuenca & 0.82 & 0.78 & 0.69 & 0.80 & 0.77 & 0.72 \\
3 & Pereira & 0.93 & 0.86 & 0.72 & 0.89 & 0.84 & 0.77 \\
4 & Snyder & 0.77 & 0.72 & 0.65 & 0.74 & 0.72 & 0.68 \\
5 & Calculado & $\mathbf{2 . 7 5}$ & 0.99 & 0.24 & 0.90 & 0.72 & 0.37 \\
\hline
\end{tabular}

El coeficiente calculado promedio se encuentra fuera del rango de los valores recomendados por NWS $(0.40$ - 0.87) y FAO (0.40 - 0.85), Allen-Pruitt, Cuenca y Sny- der se encuentran en ambos rangos, en tanto que Pereira se encuentra en el rango recomendado por NWS y fuera del rango recomendado por FAO (Tabla 2, columna 4).

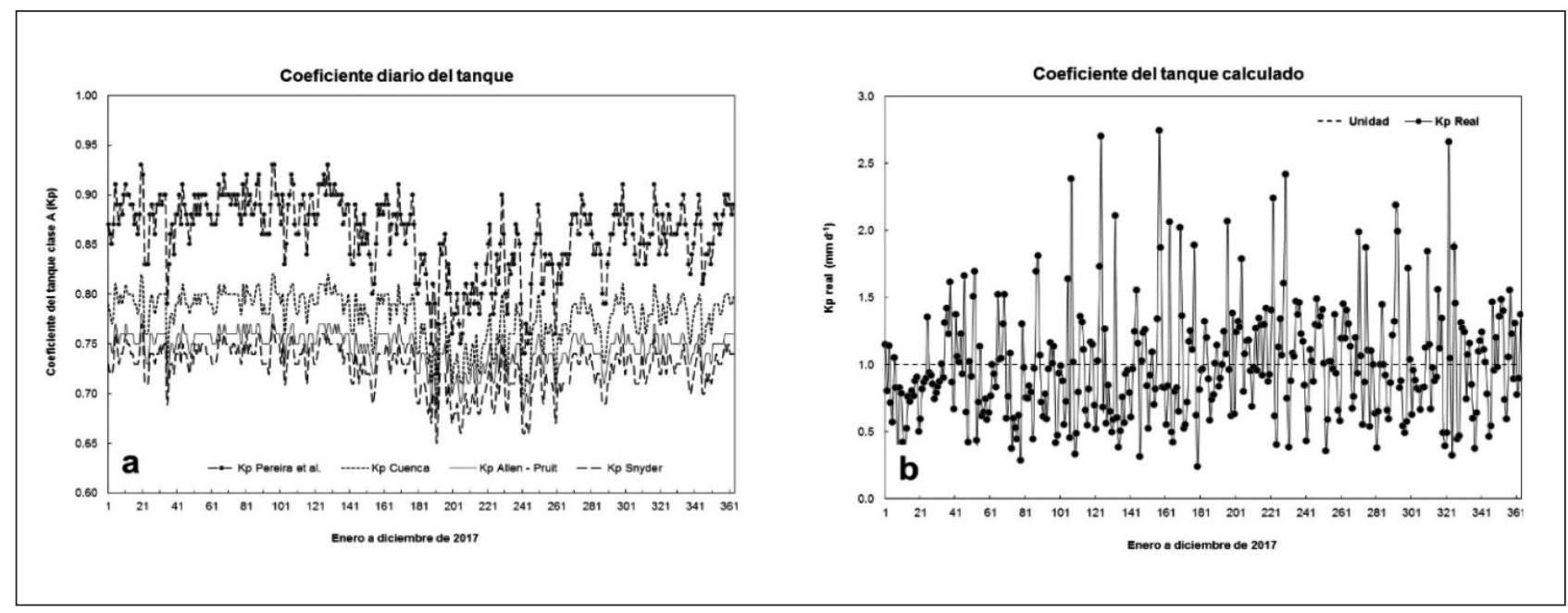

Figura 2. Coeficiente del tanque evaporímetro: (a) coeficiente determinado para los métodos en estudio, (b) coeficiente calculado.

El análisis cruzado realizado para determinar el método que proporciona el coeficiente del tanque que permita estimar la $\mathrm{ET}_{\mathrm{o}}$ diaria con una mayor precisión (con base en MAE, D y R ${ }^{2}$ ), permitió conocer que, el contraste 1 (análisis entre Tan-AP y Tan-S) proporcionó los índices de mayor precisión, siendo el contraste 13 (análisis entre FAO56 y Tan-S) el de menor precisión (Tabla 3, columnas 3, 4 y 5).

El método estándar FAO56 Penman Monteith presentó índices inferenciales bajos (Tabla 3) debido a que proporciona valores de evapotranspiración de referencia similares a la evaporación del tanque (Figura 2b).

\subsection{Coeficiente del tanque para información climatológica mensual}

El análisis cruzado realizado para determinar el método que proporciona el coeficiente del tanque que permita estimar la $\mathrm{ET}_{\mathrm{o}}$ mensual con mayor precisión (basado en MAE, $\mathrm{D}_{\mathrm{y}} \mathrm{R}^{2}$ ), a través del tanque evaporímetro permitió determinar que, el contraste 9 (análisis entre Tan-S y Tan-Kpc) proporcionó los índices de mayor precisión, siendo el contraste 11 (análisis entre FAO56 y Tan-P) el de menor precisión (Tabla 3, columnas 6, 7 y 8). 
Tabla 3. Índices inferenciales para información del clima diaria y mensual.

\begin{tabular}{|c|c|c|c|c|c|c|c|}
\hline \multirow{2}{*}{ No. } & \multirow{2}{*}{ Contraste } & \multicolumn{3}{|c|}{ Datos diarios } & \multicolumn{3}{|c|}{ Datos mensuales } \\
\hline & & MAE & $\mathrm{D}$ & $\mathrm{R}^{2}$ & MAE & $\mathrm{D}$ & $\mathrm{R}^{2}$ \\
\hline 1 & Tan-AP vs Tan-S & 0.09 & 1.00 & 1.00 & 3.48 & 0.99 & 1.00 \\
\hline 2 & Tan-AP vs Tan-C & 0.12 & 1.00 & 1.00 & 3.95 & 0.99 & 1.00 \\
\hline 3 & Tan-AP vs Tan-P & 0.44 & 0.97 & 1.00 & 14.32 & 0.93 & 0.99 \\
\hline 4 & $\begin{array}{c}\text { Tan-AP vs Tan- } \\
\text { Kpc }\end{array}$ & 1.01 & 0.83 & 0.99 & 2.86 & 1.00 & 1.00 \\
\hline 5 & Tan-C vs Tan-S & 0.21 & 0.99 & 1.00 & 7.42 & 0.98 & 1.00 \\
\hline 6 & Tan-C vs Tan-P & 0.31 & 0.98 & 1.00 & 10.38 & 0.96 & 1.00 \\
\hline 7 & Tan-C vs Tan-Kpc & 0.88 & 0.87 & 0.99 & 6.62 & 0.98 & 0.99 \\
\hline 8 & Tan-S vs Tan-P & 0.52 & 0.95 & 1.00 & 17.80 & 0.88 & 1.00 \\
\hline 9 & Tan-S vs Tan-Kpc & 1.09 & 0.79 & 0.99 & 1.82 & 0.99 & 0.99 \\
\hline 10 & Tan-P vs Tan-Kpc & 0.61 & 0.93 & 0.98 & 17.00 & 0.90 & 0.99 \\
\hline 11 & FAO56 vs Tan-P & 1.20 & 0.71 & 0.19 & 20.73 & 0.60 & 0.31 \\
\hline 12 & FAO56 vs Tan-C & 1.24 & 0.65 & 0.20 & 15.40 & 0.69 & 0.32 \\
\hline 13 & FAO56 vs Tan-AP & 1.28 & 0.62 & 0.21 & 14.73 & 0.72 & 0.33 \\
\hline 14 & FAO56 vs Tan-S & 1.31 & 0.59 & 0.20 & 14.56 & 0.73 & 0.33 \\
\hline 15 & FAO56 vs Tan-Kpc & 1.23 & 0.77 & 0.23 & 0.00 & 1.00 & 1.00 \\
\hline
\end{tabular}

El coeficiente promedio calculado mensual por los diferentes métodos (Tabla 2, columna 4) se encuentra en el rango de FAO y NWS. El coeficiente Kp calculado máximo y mínimo se encuentra fuera de los rangos FAO y NWS, mientras que el promedio se ajusta a dichos rangos, situación similar ocurre con el método Pereira; los valores proporcionados por los otros métodos se ajustan a los rangos señalados. Los resultados del coeficiente del tanque determinados en el estudio concuerdan con los obtenidos para condiciones ambientales de Sao Paulo en Brasil (Sentelhas \& Folegatti, 2003); zona sur del Uruguay (Pupo \&
García, 2009) y para algunos sitios del Perú (Hamilton, 2013).

Para el año de máxima evaporación de la serie de datos (1990), FAO56 sobreestima Tan-Kpc de noviembre a septiembre y subestima en octubre (Figura 3b). Para el año de evaporación promedio de la serie (2002), FAO56 sobreestima Tan-Kpc de enero a abril y de octubre a diciembre y subestima desde mayo a septiembre (Figura 3c). Para el año de evaporación mínima de la serie (2008), FAO56 sobreestima Tan-Kpc desde agosto hasta junio y subestima en el mes de julio (Figura 3d). 
Figura 3. Coeficiente del tanque calculado y evapotranspiración de referencia: (a) coeficiente del tanque calculado; Tan-Kpc y FAO56 para: (b) año de evaporación máxima, (c) evaporación promedio y (d) evaporación mínima.

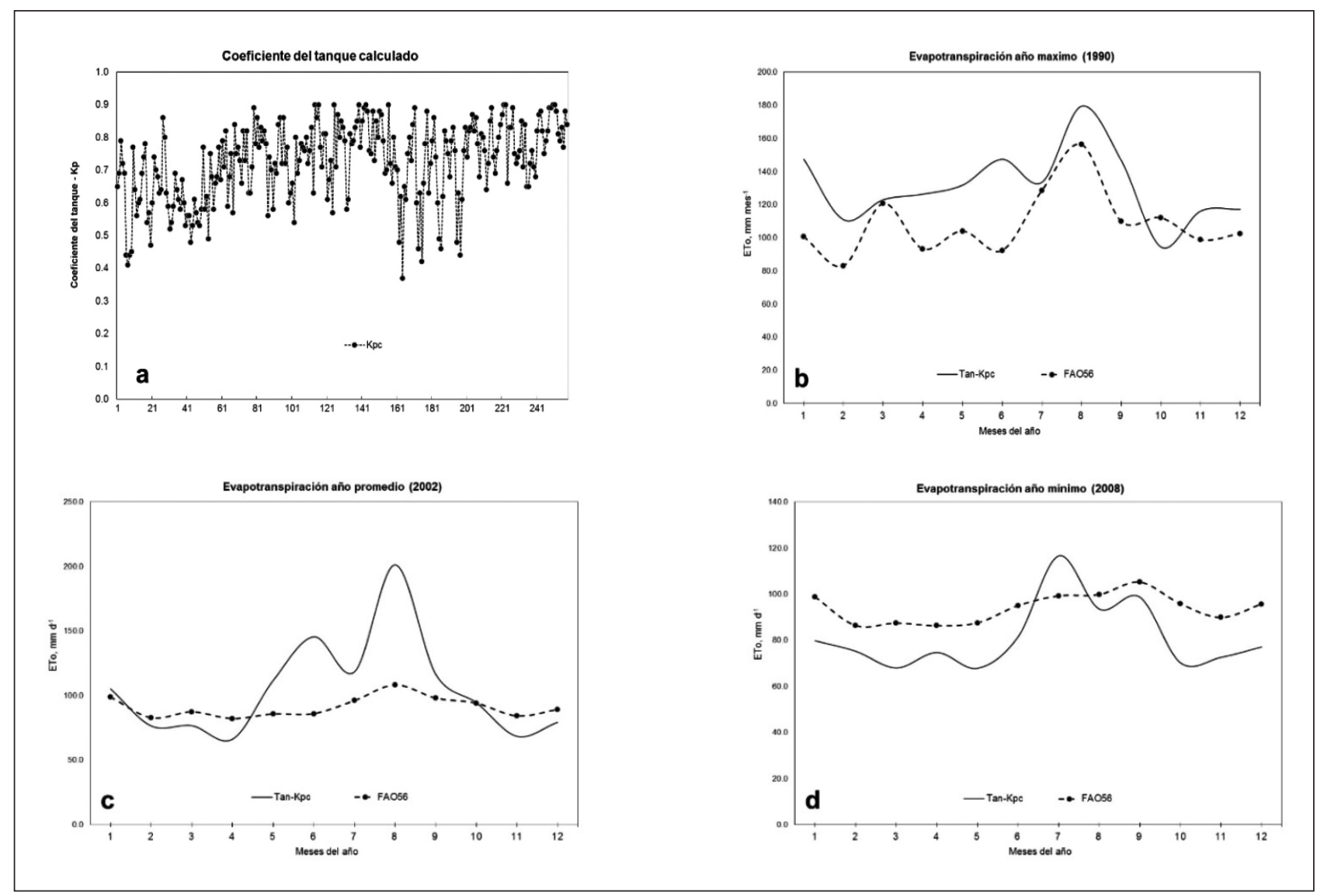

3.3 Evapotranspiración de referencia por el método del tanque evaporímetro

La evapotranspiración de referencia mensual determinada a través del tanque evaporímetro con el coeficiente del tanque calculado (Kp promedio de 0.72 ) permitió estimar el valor máximo de ET para el mes de agosto con $139.32 \mathrm{~mm}$ y el menor en abril con $76.54 \mathrm{~mm}$ (Tabla 4, columna 3).

Tabla 4. Evapotranspiración de referencia para el valle de Tumbaco.

\begin{tabular}{lcc}
\hline \multicolumn{1}{c}{ Mes } & Eo & $\begin{array}{c}\text { ET } \\
\mathrm{mm} \mathrm{mes}^{-1}\end{array}$ \\
& 130.40 & 93.89 \\
\hline Enero & 115.70 & 83.30 \\
Febrero & 113.50 & 81.72 \\
Marzo & 106.30 & 76.54 \\
Abril & 120.90 & 87.05 \\
Mayo & 143.80 & 103.54 \\
Junio & 173.40 & 124.85 \\
Julio & 193.50 & 139.32 \\
Agosto & 153.90 & 110.81 \\
Septiembre & 127.30 & 91.66 \\
Octubre & 113.60 & 81.79 \\
Noviembre & 122.10 & 87.91 \\
Diciembre & & \\
\hline
\end{tabular}




\section{Discusión}

Los dos métodos en estudio presentan diferencias fundamentales en los procedimientos matemáticos para estimar $\mathrm{ET}_{\mathrm{o}}$, además de la gran cantidad de información que requiere el modelo FAO56, se requiere calibrar localmente los parámetros a y b de la ecuación de Angstrom para estimar la radiación neta de onda corta, así como, la resistencia aerodinámica del cultivo de referencia, por otro lado, el tanque evaporímetro, a pesar de su sencillez, requiere de una operación y mantenimiento riguroso para obtener lecturas confiables de evaporación, considerando que el color del tanque, lámina de agua, dificultad para leer la evaporación durante las lluvias, entre otros factores, influyen en el balance energético y en los valores registrados. Otro factor importante a tener en cuenta es el albedo, cuyo valor para el cultivo de referencia es 0.23 y para una superficie libre de agua de 0.05 , esto deriva en que el agua almacena la radiación en un $95 \%$ en forma de calor, el cual, además del calor transferido por el tanque, produce una evaporación durante la noche, situación que no ocurre con el cultivo (Allen et al., 2006).

Para condiciones ambientales de Sao Paulo (Brasil), con base en la $\mathrm{ET}_{0}$ determinada a través de lisímetros, los métodos para calcular Kp que proporcionaron los mejores resultados de $\mathrm{ET}_{\mathrm{o}}$ fueron Pereira y Cuenca, siendo FAO56 la tercera opción para estimar ET (Sentelhas \& Folegatti, 2003); el método FAO56 presentó parámetros inferenciales de baja precisión en Costa Rica, cuyo clima es tropical (Hernández, 2014), así mismo, en Shuttleworth Wallace en los Estados Unidos tampoco proporcionó resultados confiables debido a que la resistencia aerodinámica del cultivo es menor al determinado en campo, subestimando $\mathrm{ET}_{\mathrm{o}}$ (Stannard, 1993). Los resultados encontrados en algunos lugares de la región, demuestran que FAO56 y el tanque evaporímetro deben calibrarse localmente con base en lisímetros para obtener valores de $\mathrm{ET}_{\mathrm{o}}$ confiables.

El coeficiente Kp calculado promedio diario es igual a la unidad debido a que FAO56 proporciona valores de evapotranspiración igual a la evaporación del tanque; a nivel mensual, FAO56 presentó un mejor desempeño estadístico considerando que el promedio del coeficiente del tanque se encuentra en el rango recomendado por FAO y NWS, observándose que el método FAO56 proporciona índices estadísticos de mejor precisión para información climatológica promedio mensual.
El escenario Tan-S versus Tan-Kpc presentó el mejor ajuste según los índices MAE, D y R², siendo entonces, el método Snyder y el coeficiente calculado promedio de la serie histórica los que proporcionaron los índices de mayor precisión; a pesar de que el método Snyder se desarrolló para condiciones ambientales de los Estados Unidos, éste presentó un mejor ajuste para las condiciones ecuatoriales, en donde, climatológicamente existen dos estaciones climáticas bien diferenciadas (invierno y verano); en invierno, las lluvias se presentan por el efecto atmosférico que produce la corriente cálida El Niño y en verano, el clima se encuentra afectado por la corriente fría de Humboldt, por otro lado, la cordillera oriental y occidental de los Andes también influyen en el clima, con la presencia de los nevados (Volcán Cotopaxi, Antisana, entre otros), los cuales producen temperaturas muy bajas o heladas en verano, por otro lado, en los meses de julio y agosto, la presencia de los vientos incrementa la tasa de evapotranspiración, llegando a ser ésta incluso mayor que la $\mathrm{ET}_{\mathrm{o}}$ registrada en la costa ecuatoriana.

A pesar de los problemas asociados con las lecturas de la lámina de agua evaporada desde el tanque evaporímetro, como: dificultad para leer las láminas de agua en el tanque en días lluviosos, excesiva evaporación cuando la lámina de agua en el tanque desciende a niveles críticos, transferencia de calor en función del color del tanque, almacenamiento de calor en el volumen de agua contenida en el tanque, entre otras variables, los índices inferenciales determinados con el método Snyder permiten establecer que éste método es el más confiable para estimar la evapotranspiración de referencia utilizando el método del tanque evaporímetro para las condiciones ambientales del valle de Tumbaco.

\section{Conclusiones}

El estudio permitió obtener las siguientes conclusiones:

El método estándar Penman Monteith FAO 56, para información climatológica diaria, sobreestimó $\mathrm{ET}_{\mathrm{o}}$ y presentó índices estadísticos bajos, en tanto que, para información promedio mensual presentó índices inferenciales de mayor precisión.

De los cinco métodos evaluados para determinar el coeficiente Kp, el método Snyder proporcionó índices inferenciales de mayor precisión para estimar la $\mathrm{ET}_{\mathrm{o}}$ a nivel diario y mensual, sien- 
do el coeficiente Kp promedio de 0.72 . El coeficiente $\mathrm{Kp}$ calculado promedio (0.72) de la serie histórica también puede utilizarse para determinar $\mathrm{ET}_{\mathrm{o}}$ para información climatológica mensual.

\section{Referencias}

Allen, R., Pereira, L., Raes, D., \& Smith, M. (2006). Evapotranspiración del cultivo. Roma: FAO. $300 \mathrm{p}$.

Bhabagrahi, S., Imtisenla, W., Bidyut, D., \& Bhagwati, B. (2012). Standardization of Reference Evapotranspiration Models for a Subhumid Valley Rangeland in the Eastern Himalayas. Journal of Irrigation and Drainage Engineering, 138, 880-895.

Chai, T. (2014). Root mean square error (RMSE) or mean absolute erro (MAE)?. Geoscientific Model Development Discussions, 7, 1247-1250.

Doorenbos, J., \& Pruitt, W. (1977). Guidelines for predicting crop water requirements. Roma: FAO.

Grismer, M., Orang, M., Snyder, R., \& Matyac, R. (2002). Pan evaporation to reference evapotranspiration conversion methods. Journal of Irrigation and Drainage Engineering, 128, 180-184.

Hamilton, Y. (2013). Análisis de la evapotranspiración potencial en función de elementos climáticos en la zona circunlacustre de la cuenca del Titicaca Peruano. ECIPerú, 9, 46-52.

Hernández, K. (2014). Evaluación del método FAO56 en Costa Rica. Meterorología y Oceanografia, 13, 56-67.
El valor mayor de $\mathrm{ET}_{\mathrm{o}}$ determinada a través del tanque evaporímetro con el coeficiente Kp promedio de 0.72 , se registró en el mes de agosto con $139.32 \mathrm{~mm}$ y el valor menor en abril con $76.54 \mathrm{~mm}$

Pereira, A., Villa, N., Pereira, A., \& Barbieri, V. (1995). A model for the class A pan coefficient. Agricultural and Forest Meteorology, 76, 75-82.

Pupo, L., \& García, P. (2009). Estimación de los coeficientes de tanque "A" para calcular la evapotranspiración del cultivo de referencia en la zona sur del Uruguay. Agrociencia, 13, 47-51.

Samuels, P. (2014). Pearson Correlation. Disponible en: http://www.statstutor.ac.uk/resources/ uploaded/pearsoncorrelation3.pdf

Sentelhas, P., \& Folegatti, M. (2003). Class A pan coefficients $(\mathrm{Kp})$ to estimate daily reference evapotranspiration (ET ET $_{\mathrm{o}}$. Revista Brasileira de Engenharia Agricola e Ambiental, 7, 111-115.

Snyder, R., Orang, M., Matyac, S., \& Grismer, M. (2005). Simplified Estimation of Reference Evapotranspiration from Pan Evaporation Data in California. Journal of Irrigation and Drainage Engineering, 131, 249-253.

Stannard, D. (1993). Comparison of Penman-Monteith, Shuttleworth-Wallace, and Modified Priestley-Taylor Evapotranspiration Models for wildland vegetation in semiarid rangeland. Water Resources Research, 29, 1379-1392.

Willmott, C., Robeson, S., \& Matsuura, K. (2011). A refined index of model performance. International Journal of climatology, 32, 2088-2094. 